\title{
Asociación entre las teorías implícitas sobre la enseñanza, el aprendizaje y la acción de problematizar
}

\section{Association between the implicit theories on teaching and learning, and the act of problematizing}

\author{
Carlos Fernando Mariño \\ Magíster en Educación \\ Corporación Universitaria Minuto de Dios \\ Bucaramanga, Colombia \\ Correo electrónico: carlos.marino@ustabuca.edu.co
}

Recibido 22 de julio de 2016; aceptado 18 de septiembre de 2016

\section{Cómo citar este artículo:}

Mariño, C.F. (2017). Asociación entre las teorías implícitas sobre la enseñanza, el aprendizaje y la acción de problematizar. Espiral, Revista de Docencia e Investigación, 7(1), 103 - 128

\section{Resumen}

Objetivo. El objetivo del presente artículo de investigación es verificar la existencia de una asociación entre las teorías implícitas de los docentes sobre la enseñanzaaprendizaje y la acción de problematizar. Entendida esta como la estrategia didáctica en la que los estudiantes son problematizados. Se considera que existen, en este dominio, tres tipos de teorías implícitas: directa, interpretativa y constructiva, de acuerdo con la exposición de Juan Ignacio Pozo y colaboradores. En la acción de problematizar se han delimitado sus características a partir de los aportes de Jhon Dewey, Paulo Freire y Demerval Saviani.

Metodología. Para lograr el objetivo de la investigación se diseñó un estudio transeccional correlacional en el que se analizó la existencia de una correlación estadísticamente significativa entre las variables consideradas, en una muestra no probabilística, compuesta por 15 docentes de un programa de psicología.

Resultados. Los resultados obtenidos mediante el coeficiente de correlación rho de Spearman no permiten afirmar la existencia de tal asociación. Aunque es significativo que en la misma muestra prevalezca la teoría implícita constructiva junto con la alta implementación de la acción de problematizar.

Palabras clave: Teorías implícitas, acción de problematizar, problema, problematización, enseñanza, aprendizaje.

\section{Abstract}

objective. The objective of this research article is to verify the existence of an association between the implicit theories of teachers on teaching-learning and the action of problematize, understanding as the didactic strategy in which students are problematizados. It is considered that there are three types of implicit theories in this domain: direct, interpretative and constructive, according to the exhibition by Juan Ignacio Pozo and collaborators. In the action of problematizing the characteristics have been taken from the contributions of Jhon Dewey, Paulo Freire and Demerval Saviani.

Methodology. In order to achieve the objective of the research, a correlational transactional study was designed to analyse the existence of a statistically significant correlation between the variables considered, in a non-probabilistic sample, composed of 15 teachers of a psychology program.

Results. The results obtained, by means of the coefficient of correlation rho of Spearman, do not allow to affirm the existence of such association. Although it is significant that in the same sample prevails the constructive implicit theory along with the high implementation of the action of problematize.

Keywords: Implicit theories, problematize action, problem, problematization, teaching, learning. 


\section{Introducción}

En esta investigación se consideró la posible relación entre las teorías implícitas de los docentes sobre la enseñanza y el aprendizaje y la acción de problematizar. Las diversas actividades de los profesores están condicionadas por sus pensamientos implícitos sobre los diversos dominios de su actividad. Es importante, entonces, considerar si las teorías implícitas de los docentes están asociadas a su actividad, específicamente a la acción de problematizar, que enfatiza la activación de la problematización en el proceso de enseñanza y aprendizaje.

El objetivo principal de este estudio es ver si hay relación entre la acción de problematizar, por parte de los docentes, y los tipos de teorías implícitas sobre la enseñanza y el aprendizaje que estos tienen.

Se plantean además los siguientes objetivos específicos:

1. Evaluar el grado en que cada una de las teorías implícitas sobre la enseñanza y el aprendizaje está presente en cada uno de los docentes que participan en la investigación.

2. Elaborar un instrumento de evaluación de la acción de problematizar efectuada por parte de los docentes.

3. Evaluar grado de realización de la acción de problematizar.

El objetivo principal corresponde al problema de investigación que se expone en la siguiente pregunta: ¿Existe una relación entre la acción de problematizar y los tipos de teorías implícitas sobre la enseñanza y el aprendizaje utilizados por los docentes del programa de Psicología?

\section{Teorías implícitas sobre la enseñanza y el aprendizaje}

Diferentes enfoques teóricos y metodológicos han abordado las concepciones de profeso- res y alumnos sobre la enseñanza y aprendizaje. Al respecto Pérez, Mateos, Scheuer \& Martín (2006) reportan los siguientes: metacognición, teoría de la mente, creencias epistemológicas, fenomenografía, teorías implícitas y el perfil del docente y análisis de la práctica. Este último, aunque no es un enfoque, se refiere a un conjunto de investigaciones que influyen en la tarea del profesor en las diversas fases de su actividad.

A partir del enfoque sobre las teorías implícitas las concepciones del aprendizaje son entendidas como representaciones complejas. Pérez et al. (2006) aseveran que ellas constituyen "un conjunto de principios que restringen tanto nuestra forma de afrontar como de interpretar o entender las distintas situaciones de enseñanza-aprendizaje las que nos enfrentamos" ( $p$. 79). Las concepciones, por tanto, constituyen auténticas teorías, no simples ideas aisladas, las cuales, por ser implícitas, no son accesibles a la conciencia, aunque sí lo puedan ser sus productos.

Las teorías implícitas del profesor corresponden a un enfoque dentro del paradigma de estudio de la actividad docente, conocido como el pensamiento del profesor (Rodrigo, Rodríguez \& Marrero, 1993). Según Pérez (1983), las investigaciones enmarcadas en el pensamiento del profesor, cuyo origen se data en las décadas de los 60 y 70 del siglo XX, en lugar de centrar la atención en las consecuencias de las actitudes de los profesores, propio del paradigma denominado "proceso-producto" o "presagioproducto", se concentran en los motivos que explican las diversas actuaciones de los profesores. Se pasa del énfasis en el comportamiento observable al énfasis en los procesos cognitivos que sustentan las intenciones y acciones del profesor.

De acuerdo con el enfoque socio-constructivista las teorías implícitas son representaciones individuales basadas en experiencias sociales y culturales (Rodrigo et al., 1993). Respecto a los 


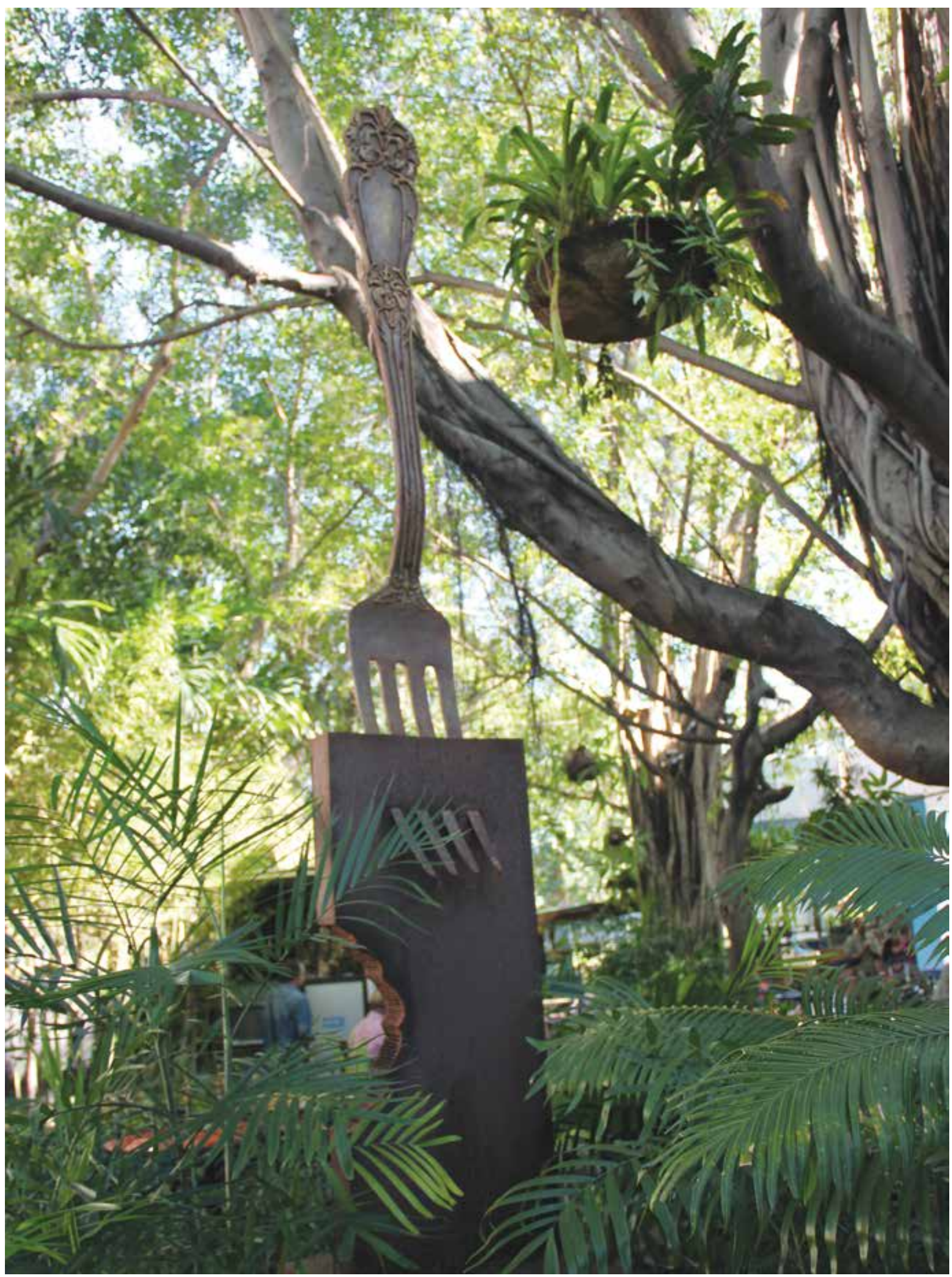


profesores, tales cogniciones se refieren a los diversos aspectos de su quehacer profesional: enseñanza y aprendizaje, evaluación, etc., que implican las experiencias biográficas directas, vicarias o simbólicas, basadas en situaciones teóricas, prácticas, afectivas, morales, personales o sociales.

Las teorías implícitas funcionan como verdaderas creencias que sirven como punto estable y eficaz para interpretar situaciones, tomar decisiones rápidas, en la cotidianidad. Estas tienen un origen y una transmisión socio cultural. Aunque son individuales, se deben al contacto con un contexto sociocultural. Están mediadas por prácticas culturales y formas sociales de interacción. De este modo, individual y social acontecen de manera dialéctica en un contexto cultural, caracterizado por ser un conjunto de prácticas, actividades y formas de interacción regulares (Rodrigo et al., 1993).

La presentación de las teorías implícitas sobre la enseñanza y el aprendizaje sigue la exposición de Pozo, Scheuer, Mateos, Pérez \& Echeverria (2006) en la que formulan las teorías implícitas de profesores y alumnos acerca de la enseñanza y aprendizaje. Estas son: la teoría directa, la teoría interpretativa, la teoría constructivista y la teoría posmoderna. El trabajo de Pozo y sus compañeros recoge más de una década de investigaciones sobre el asunto; además, en la revisión bibliográfica aparece como el autor que se ocupa explícitamente de este dominio de las teorías implícitas.

Pozo et al. (2006) explican que el estudio y formulación de las teorías implícitas de los profesores y alumnos acerca de la enseñanza y aprendizaje se ha hecho a partir del análisis del aprendizaje, entendido como un sistema en el que se relacionan tres componentes principales: las condiciones, los procesos del aprendiz y los resultados. Las condiciones se refieren a los diferentes aspectos que caracterizan al aprendiz y su entorno. Los procesos incluyen las acciones, manifiestas o mentales, que se realizan al aprender. Los resultados corresponden a lo que se aprende o se pretende aprender.

Los estudios realizados apuntan a tres teorías básicas que median la relación con la enseñanza y aprendizaje. Estas reciben el nombre de teoría directa, teoría interpretativa y teoría constructiva. Sin embargo, algunos trabajos incluyen una cuarta posición, llamada posmoderna, cuya condición de teoría implícita es incierta, por este motivo no será tenida en cuenta al momento de verificar su presencia en los docentes que participan en la investigación.

En la tabla 1 se exponen las teorías mencionadas, según los componentes principales propuestos por Pozo et al. (2006). La definición de cada tipo de teoría explicita sobre la enseñanza y el aprendizaje resulta útil, pues permite analizar la relación existente entre las concepciones de los docentes sobre los diversos componentes del aprendizaje y la ejecución explícita de la didáctica de la que se trata en esta investigación.

La teoría directa se basa en un realismo ingenuo, en cuyo caso hay una correspondencia directa y univoca entre el conocimiento y la realidad. De esto se deriva un dualismo epistemológico: el conocimiento es verdadero o es falso. En el primer caso es copia fiel de la realidad, en el segundo, no corresponde con ella. En la versión extrema aprender equivale a los resultados. En las versiones más elaboradas, se incluyen las condiciones automáticas y contingentes del mismo. Por tanto, ontológicamente, el aprendizaje es un estado o proceso aislado (Pozo et al., 2006).

Desde el punto de vista epistemológico, la teoría interpretativa es igualmente realista, pues en últimas conocer corresponde a reflejar la realidad. El aprendizaje se presenta como un proceso, que en su sentido ontológico equivale a hablar de una entidad que ocurre a lo largo del tiempo. En lo conceptual, la teoría se articula en tres componentes básicos el aprendizaje 
Tabla 1. Teorías implícitas del aprendizaje y sus componentes principales

\begin{tabular}{|c|c|c|c|}
\hline Teoría & Condiciones & Procesos & Resultados \\
\hline Directa & $\begin{array}{l}\text { No se preocupa con esta } \\
\text { dimensión. Estas, en una } \\
\text { versión sofisticada, se } \\
\text { entienden como contingencias } \\
\text { que facilitan u obstaculizan el } \\
\text { aprendizaje. }\end{array}$ & $\begin{array}{l}\text { En la versión extrema no se } \\
\text { preocupa siquiera de esta } \\
\text { dimensión. }\end{array}$ & $\begin{array}{l}\text { Supone que el resultado se garantiza } \\
\text { con la simple exposición al contenido } \\
\text { u objeto de aprendizaje. } \\
\text { Se conciben como productos } \\
\text { claramente identificables y } \\
\text { cuantificables. Corresponden a } \\
\text { copias fieles de la realidad o modelo } \\
\text { percibido. }\end{array}$ \\
\hline Interpretativa & $\begin{array}{l}\text { Son necesarias para el } \\
\text { aprendizaje; aunque } \\
\text { insuficientes para explicarlo. } \\
\text { La condición básica en la } \\
\text { propia actividad del aprendiz, } \\
\text { que puede ser fuente de } \\
\text { distorsión. De ahí que se } \\
\text { intervenga en su actividad } \\
\text { con el fin de lograr una } \\
\text { apropiación más fiel del objeto } \\
\text { de aprendizaje. }\end{array}$ & $\begin{array}{l}\text { Los procesos mentales del aprendiz } \\
\text { y la regulación de las propias } \\
\text { prácticas cumplen una función } \\
\text { explicativa del aprendizaje. }\end{array}$ & $\begin{array}{l}\text { Como en la teoría directa son una } \\
\text { copia de la realidad. } \\
\text { En algunas ocasiones se entienden } \\
\text { como acumulación de conocimientos. } \\
\text { También como incremento en la } \\
\text { complejidad de los conocimiento } \\
\text { preexistentes. }\end{array}$ \\
\hline
\end{tabular}

Se deben considerar los cambios en los procesos representacionales del

Constructiva aprendiz, su manera de dar significado al objeto de aprendizaje y las metas de aprendizaje que se propone.
Implica procesos mentales que reconstruyen las representaciones de la realidad, incluso a sí mismas, y que regulan la actividad de aprender.
Constituyen una redefinición de los contenidos u objetos de aprendizaje, así como de la persona que aprende.

Fuente: Elaboración propia.

es secuencial, escalonado y unidireccional. Las condiciones actúan en el proceso del aprendiz, que por su parte dan lugar al aprendizaje. Estos resultados son la base, escalón de posteriores aprendizajes (Pozo et al., 2006).

El rasgo distintivo de la teoría constructiva es la base epistemológica, según la cual se puede atribuir diversos significados a un mismo contenido, el cual es asumido de modos diversos, por las distintas personas. Esto da lugar a diversos grados de incertidumbre respecto al conocimiento, cuya adquisición necesita de la transformación de lo que se aprende y del aprendiz. Esta transformación puede dar lugar a una innovación del conocimiento cultural. En lo referente a los componentes ontológico y conceptual, esta teoría concibe el aprendizaje como un sistema autorregulado compuesto por condiciones, procesos y resultados. Es im- portante notar que la referencia a la influencia de los procesos previos en el aprendizaje no basta para que el pensamiento de un profesor sea constructivo, se debe asumir que este y los procesos que los preceden son fruto de una construcción (Pozo et al., 2006).

Cada una de estas teorías implícitas sobre la enseñanza y el aprendizaje equivale a una diversa manera de concebir qué es aprender, cómo se aprende, qué se aprende, $y$, en consecuencia, qué y cómo se evalúa. Así, en la teoría constructiva se privilegia el aprendizaje de estrategias sobre el aprendizaje de contenidos conceptuales, más apropiados para la teoría directa e interpretativa. Respecto a cómo se aprende, la teoría directa enfatizará la memorización, mientras la teoría directa se refiere a la incorporación de los contenidos a partir de diversos medios, mediante capacidades cogni- 
tivas complejas, y la teoría constructiva recalcará la atribución de significados a los diversos contenidos.

\section{La acción de problematizar}

En esta investigación se utiliza la expresión acción de problematizar en lugar de problematización, pues se quiere enfatizar la acción que intencionalmente actúa un sujeto, en este caso los docentes (profesoras o profesores). Sin embargo, esta actividad corresponde a la concepción que se tenga de problema y problematización, en el contexto de la enseñanza y el aprendizaje.

Según Orange (2005) el problema se incluye en el proceso de enseñanza y aprendizaje, porque es así como proceden los investigadores y porque es una oportunidad para quebrar las preconcepciones de los estudiantes. Por su parte Fabre (2005) tras considerar los aportes de Dewey y Bachelard, sobre el problema en la generación de conocimiento, sostiene que estos autores enfatizan la construcción del problema, antes que su resolución del mismo. Si se considera que el conocimiento no es fetiche que se expone a los alumnos, sino una adquisición, entonces se requiere el problema, la problematización, para que surja el conocimiento.

En el estudio de Orange (2005), sobre experiencias llevadas a cabo por docentes, se aprecia una gran diversidad conceptual y metodológica del problema y la problematización. Según esto, la acción de problematizar, como actividad de los docentes en la que confrontan a los estudiantes con el problema, tiene múltiples formas de realización. Con todo, los diversos teóricos de la problematización coinciden en la concepción de conocimiento como construcción llevada a cabo mediante la confrontación con problemas, la discusión y la reflexión.

En esta investigación se asume la definición de acción de problematizar propuesta por Zanotto \& Rose (2003), que consiste en saber rela- cionar de forma coherente y secuenciada (1) la identificación de un problema, (2) la búsqueda de los factores que lo explican y (3) la generación de soluciones. Estas autoras proponen esta definición a partir de los aportes de John Dewey, Dermeval Saviani, Paulo Freire y de los aportes de la psicología cognitiva.

Mariño (2015) reconoce seis características de la acción problematizar, que se presentan a continuación:

1. La acción de problematizar implica una secuencia de pasos, los mismos mencionados por Zanotto \& Rose (2003).

2. El estudiante juega un papel activo. Esta característica según expone Fabre (2006) sobresale en la postura de Dewey, el problema corresponde a una situación del mundo que afecta al sujeto y requiere de este una resolución.

3. Saviani (1996) insiste en la problematicidad del problema. Esto es, no cualquier situación constituye un problema, lo que la hace tal es el sujeto que ante esta tiene que encontrar una solución, pues no puede permanecer ahí. El problema corresponde a una situación marcada por la necesidad. En tal sentido, la pregunta por sí misma no es un problema, ni el enigma o el misterio. El problema tiene un carácter vital y dramático, se trata de una situación de impase a la que se debe encontrar una solución.

4. Freire $(1983 ; 2006)$ resalta la praxis, como dimensión ética de la acción, vinculada a la resolución de problemas. El reconocimiento de una situación problema implica una postura ética, una valoración. El sujeto cognoscente está situado históricamente, de ahí que el problema además de un asunto cognitivo, es un asunto que toca a su manera de existir, de estar en relación con los otros seres humanos y su entorno. 
5. A partir de la participación activa del sujeto cognoscente no solo se construye el conocimiento, ocurre el aprendizaje, sino que se adquieren estrategias de aprendizaje. Según Ormrond (2008) se activan procesos cognitivos que se refieren a los procesos de conocimiento que hacen posible el aprendizaje, a lo cual se da el nombre de metacognición. Dado que la acción de problematizar implica precisamente la participación activa de los estudiantes, esta actividad necesariamente implica la concurrencia de procesos metacognitivos.

6. Chadwick (2001) y Ormrond (2008) consideran que a partir del cognitivoconstructivista aprender corresponde a un proceso en el que el sujeto construye la comprensión de sí mismo y de su entorno. Tal posición se basa, a su vez, en los aporte de Balwin, Dewey, Piaget, Vygotski, Bruner, entre otros, considerados precursores del enfoque cognitivoconstructivista. Según este enfoque el carácter activo del sujeto que aprende, además de implicar procesos metacognitivos, a los que ya se hizo referencia, tiene como resultado el cambio cognitivo. Según Ausubel, Novak \& Hanesian (2006) se transforma la estructura de conocimiento organizada en cada sujeto.

\section{Metodología}

El tipo de investigación propuesto permitió verificar la hipótesis en que se afirma la existencia de una correlación significativa entre el tipo y grado de teoría implícita sobre la enseñanza y el aprendizaje que caracteriza cada docente y el grado de actuación de la acción de problematizar realizada por cada uno. La investigación se llevó a cabo bajo el diseño no experimental en la modalidad transeccional correlacional.

La población objeto de estudio corresponde a los profesores del programa de Psicología, en
Bogotá, Colombia. Además, es necesario considerar la población de estudiantes, pues son ellos quienes valoran la acción de problematizar llevada a cabo por los profesores. Por tal motivo se tiene una muestra de docentes y otra de estudiantes.

Muestra de docentes. El programa de Psicología estaba compuesto, en el primer semestre de 2013, por 52 docentes (hombres y mujeres). Estos asumían las asignaturas correspondientes a las diversas áreas disciplinares y aplicadas de la psicología, correspondientes a 45 asignaturas, distribuidas en 331 grupos. Para la presente investigación se ha seleccionado una muestra a conveniencia, no probabilística, correspondiente a los docentes que aceptaron participar en la investigación, compuesta por 15 (hombres y mujeres), que se encargan de 30 grupos.

Muestra de estudiantes. Los 30 grupos a cargo de los profesores que aceptaron participar en la investigación equivalen a 625 estudiantes. Se puede deducir, se trata de una muestra no probabilística, establecida en razón de la vinculación de los estudiantes a los grupos a cargo de los docentes estudiados.

El instrumento correspondiente a las teorías implícitas sobre la enseñanza y el aprendizaje. La información correspondiente a la frecuencia con que se presenta cada teoría implícita sobre la enseñanza y el aprendizaje se obtendrá mediante el cuestionario de dilemas sobre la enseñanza y el aprendizaje, adaptado por Vilanova, García \& Señorino (2007) (apéndice 1). Los autores del cuestionario reportan que la confiabilidad del instrumento se estableció mediante el Coeficiente Alpha de Cronbach que es uno de los elementos estadísticos más usados y que se refiere a la consistencia interna de cuestionarios o escalas. Se obtuvo 0.7055 , puesto que el cuestionario consta de doce dilemas y este coeficiente depende tanto del número de reactivos, como de sus covariancias, se puede considerar que el Alpha de Cronbach es aceptable y el instrumento fiable (Vilanova et al., 2007). 
Respecto a la validez del constructo, el cuestionario da cuenta de las tres teorías implícitas mencionadas. Esta se estableció mediante un método estadístico multivariado. Específicamente realizaron un análisis de los principales componentes con rotación Varimax. Los resultados obtenidos muestran la presencia de tres dimensiones principales, estás corresponden a los tres aspectos principales abordados por la teoría.

Instrumento correspondiente a la acción de problematizar. Para evaluar la acción de problematizar se ha elaborado un cuestionario ad hoc. Se optó por construir un instrumento compuesto por reactivos representativos de cada una de las características correspondientes a la acción de problematizar.

Elaboración de un cuestionario. Los ítems se generaron a partir de las actividades que constituyen la acción de problematizar, según se aprecia en la tabla 2. Para la calificación de cada ítem se escogió una escala tipo Likert. Las opciones de respuesta, con su respectiva codificación son las siguientes: en total desacuerdo $=1$, en desacuerdo $=2$, de acuerdo $=3$, totalmente de acuerdo $=4$.

\section{Tabla 2. Ítems del cuestionario para evaluar la acción de problematizar}

\section{Características}

1. Énfasis en los tres momentos de la acción de problematizar: identificar problemas, buscar factores explicativos y proponer soluciones.

El alumno es considerado sujeto activo que construye conocimiento.

\section{Ítems}

1. El profesor promueve que los alumnos identifiquen asuntos que requieren la búsqueda de una solución.

2. El profesor promueve que los alumnos busquen información para comprender los asuntos que aparecen problemáticos.

3. El profesor promueve que los alumnos propongan soluciones a problemas sobre los cuales han investigado.

1. El profesor promueve que los alumnos usen sus conocimientos para abordar los problemas relacionados con los temas de estudio.

2. El profesor promueve que los alumnos busquen información por su propia cuenta.

3. El profesor da lugar a que se debatan los conocimientos que los alumnos han adquirido sobre los temas estudiados.

1. El profesor da lugar a actividades en las que los alumnos reconocen situaciones que necesitan ser solucionadas.

3. El problema corresponde a una situación de impase que requiere una solución por parte del sujeto.

2. Los asuntos tratados en clase hacen que usted se sienta en la necesidad de buscar información y dar alguna solución.

3. Usted considera que debe reunir información y trabajar para dar solución a los asuntos que han aparecido en la asignatura, porque si no lo hace un problema importante para su vida se deja de lado.
4. La praxis, como dimensión ética de la acción: problema y la solución tienen una dimensión social y ética.

5. La adquisición de estrategias de aprendizaje, se reflexiona sobre el propio proceso y se perfecciona la acción.

6. Implica cambio conceptual, el proceso de reflexión y problematización produce cambios cognitivos se adquieren conceptos, se reformulan o eliminan.
1. El profesor les hace caer en la cuenta de las repercusiones sociales de los problemas y soluciones propuestas.

2. Los problemas vistos en la materia son importantes para mi contexto social.

3. Al proponer soluciones a los problemas abordados en la asignatura se considera a quienes se afecta con ello.

1. Considero que he mejorado en la búsqueda de soluciones a partir de mis estrategias de aprendizaje desarrolladas en la asignatura.

2. El reconocimiento de problemas y la búsqueda de una solución me hacen reflexionar sobre mi proceso de aprendizaje.

3. Debido al reconocimiento de problemas y el trabajo de investigación para darles solución he mejorado mi manera de aprender.

1. A partir de las actividades desarrolladas en la asignatura he adquirido nuevos conceptos.

2. Las actividades desarrolladas en esta asignatura me han llevado a transformar mis conocimientos.

3. Reconozco que algunos conocimientos que traía al inicio de la asignatura no son suficientes para responder a las problemáticas que se me presentan.

Fuente: Elaboración propia. 
La prueba piloto se aplicó a 127 estudiantes, en grupos de 20 a 30 personas, vinculados a las asignaturas de tres docentes del programa de Psicología. En el análisis de la fiabilidad del instrumento, utilizando el Coeficiente Alpha de Cronbach, se obtuvo un valor de 0,87 , según el cual el instrumento se considera fiable. Para la determinación de la validez del constructo se utilizó el análisis factorial y en el Kaiser Meyer Olkin - KMO coeficiente de funcionalidad para aplicar a los datos análisis factorial se obtuvo un valor de 0,79, que indica la factibilidad del análisis factorial.

Para la verificación de la concordancia entre los factores encontrados y los supuestos teóricos se utilizó un método estadístico multivariado, específicamente el análisis de princi- pales componentes con rotación Varimax. Los resultados obtenidos muestran la presencia de cinco factores principales, como se aprecia en la tabla 3.

En la administración no se detectaron dificultades de tipo semántico, ni en la comprensión de los ítems, ni en las instrucciones. Aunque el análisis de los factores no corresponde exactamente a las características previstas, se decide conservar los reactivos, pues la prueba en conjunto se refiere a un único fenómeno. En este caso, tras la recolección total de los datos de la investigación se debe hacer un nuevo análisis factorial con el fin de determinar la constitución de los componentes y la coincidencia con las características previstas.

Tabla 3. Análisis de los principales componentes con rotación Varimax

\begin{tabular}{|c|c|c|c|c|c|c|}
\hline \multirow[t]{2}{*}{ Característica } & \multirow[t]{2}{*}{ Reactivos } & \multicolumn{5}{|c|}{ Componente } \\
\hline & & 1 & 2 & 3 & 4 & 5 \\
\hline \multirow{3}{*}{$\begin{array}{c}\text { Énfasis en los tres momentos de la acción de } \\
\text { problematizar. }\end{array}$} & 1 &, 561 & & ,444 & & \\
\hline & 17 & & ,461 & & & \\
\hline & 3 & ,476 & & ,596 & & \\
\hline \multirow{3}{*}{ El alumno es considerado sujeto activo. } & 4 & ,766 & & & & \\
\hline & 6 & & 731 & & & \\
\hline & 14 & & & & & ,730 \\
\hline \multirow{3}{*}{ Problema como situación de impase } & 12 & & & & ,647 & \\
\hline & 9 & & & 720 & & \\
\hline & 7 & & ,589 & & & \\
\hline \multirow{3}{*}{ La praxis como dimensión ética de la acción. } & 8 & & 680 & & & \\
\hline & 10 & & 756 & & & \\
\hline & 11 & & ,405 & & & 455 \\
\hline \multirow{3}{*}{ Adquisición de estrategias de aprendizaje. } & 13 & & & & ,646 & \\
\hline & 5 & ,636 & & & & \\
\hline & 15 & & &, 509 & ,446 & \\
\hline \multirow{3}{*}{ Cambio conceptual. } & 16 & ,462 & & & & ,664 \\
\hline & 2 & ,756 & & & & \\
\hline & 18 & & & & ,703 & \\
\hline
\end{tabular}

Fuente: Elaboración propia. 


\section{Resultados}

\section{Sobre las teorías implícitas de la enseñanza y el aprendizaje}

A continuación se presentan los resultados correspondientes a la aplicación del cuestionario de dilemas de Vilanova, García y Señorino (apéndice 1). Estos corresponden a la variable teorías implícitas sobre la enseñanza y el aprendizaje. En este mismo apartado se hace el análisis descriptivo de los resultados correspondientes a esta variable.

La figura 1 muestra la distribución de frecuencias de las respuestas de los profesores, discriminada por cada teoría implícita del aprendizaje considerado en cada dilema del cuestionario. Según los datos obtenidos se tiene que los docentes prefieren la teoría constructiva, seguida de la teoría interpretativa, con escasa preferencia de la teoría directa.

Según Vilanova et al. (2007) el cuestionario de dilemas aplicado permite discriminar la representación de cada una de las teorías implícitas respecto a las preguntas ¿qué es aprender?, ¿cómo se aprende? y ¿qué se aprende? La primera cuestión subyace en los dilemas 1, 6, 9 y 11. La segunda, en 5, 7, 8 y 10 . La tercera, en 2,3 y 4 .
La figura 2 muestra los promedios correspondientes a cada teoría, de acuerdo con las tres cuestiones mencionadas. Respecto a ¿qué es aprender?, entre 60 posibilidades las teorías interpretativa y constructiva están igualmente presentes, la diferencia es de 1, superando ampliamente a la teoría directa que obtuvo 5 respuestas. En la cuestión ¿cómo se aprende? sobresale la teoría constructiva, que enfatiza los procesos mentales que reconstruyen las representaciones de la realidad y que regulan la actividad de aprender. En relación con ¿qué se aprende? la teoría más elegida es la teoría constructiva, lo cual indica la preferencia del aprendizaje de estrategias, en lugar de contenidos explícitos como se supone en las otras teorías.

La figura 3 muestra la distribución de frecuencias de las respuestas de los 15 docentes, discriminada por cada teoría implícita del aprendizaje. Según los datos obtenidos se tiene que los docentes prefieren la teoría constructiva, seguida de la teoría interpretativa, con escasa preferencia de la teoría directa. De 15 docentes, 9 presentan mayor presencia de la teoría constructiva. En el docente 12 se da un empate entre la teoría interpretativa y la constructiva. Tres docentes tienen mayor presencia de la teoría directa. Se trata de los docentes 1, 7 y 11.

Figura 1. Polígono de frecuencia de las respuestas, según dilemas.

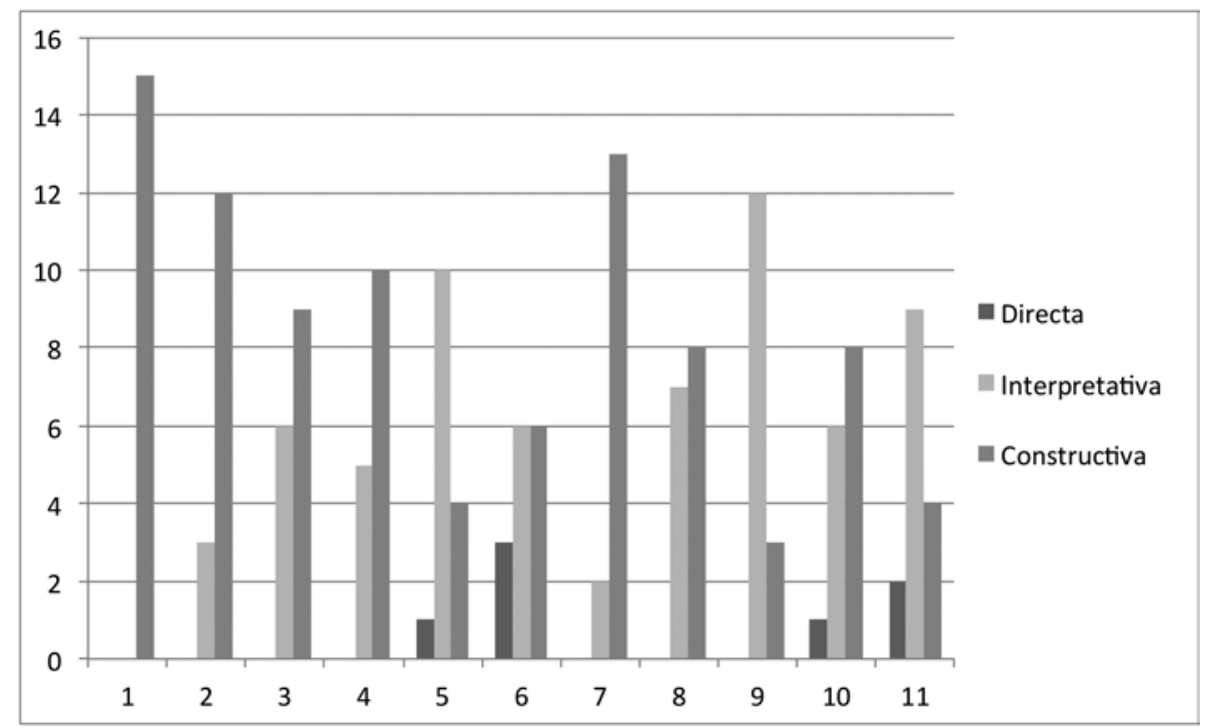


Figura 2. Polígono de frecuencia de las dimensiones ¿qué es aprender?,

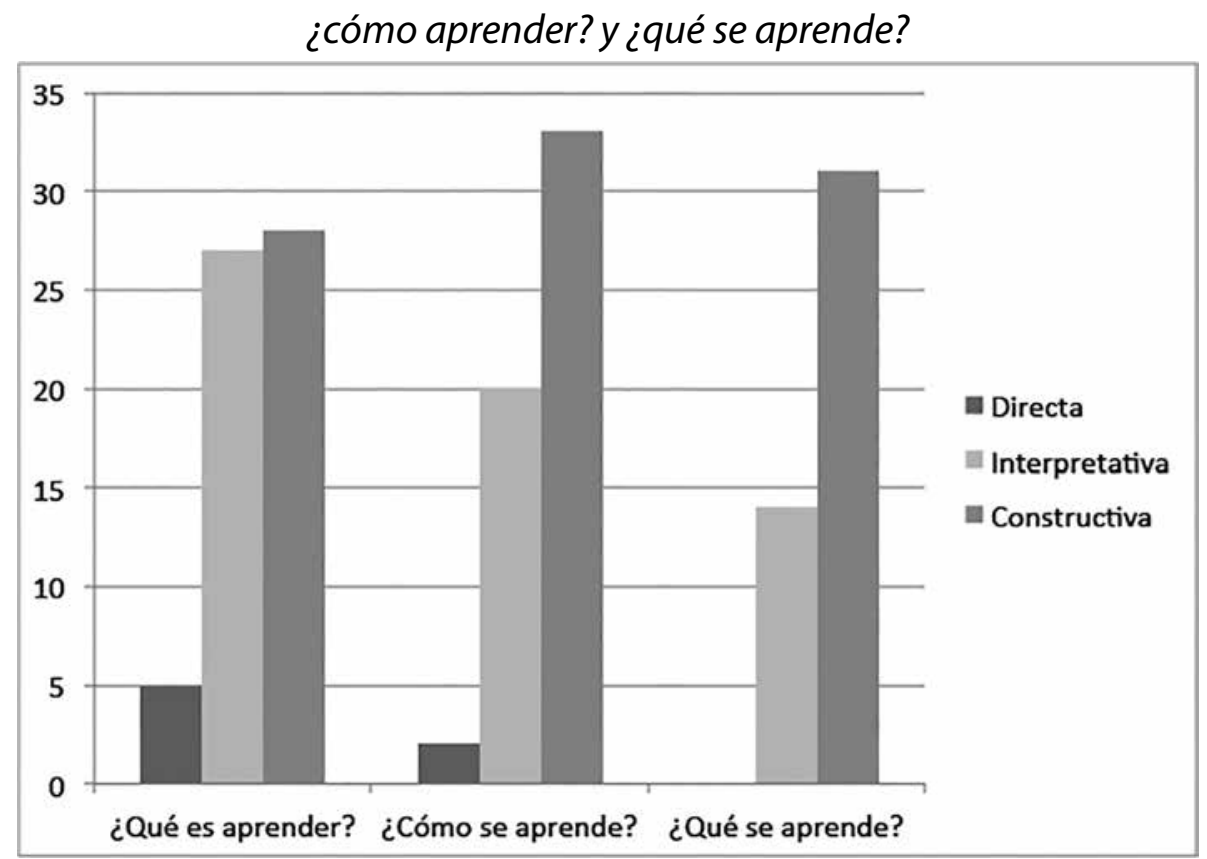

Figura 3. Polígono de frecuencia sobre la presencia de las teorías implícitas en cada docente.

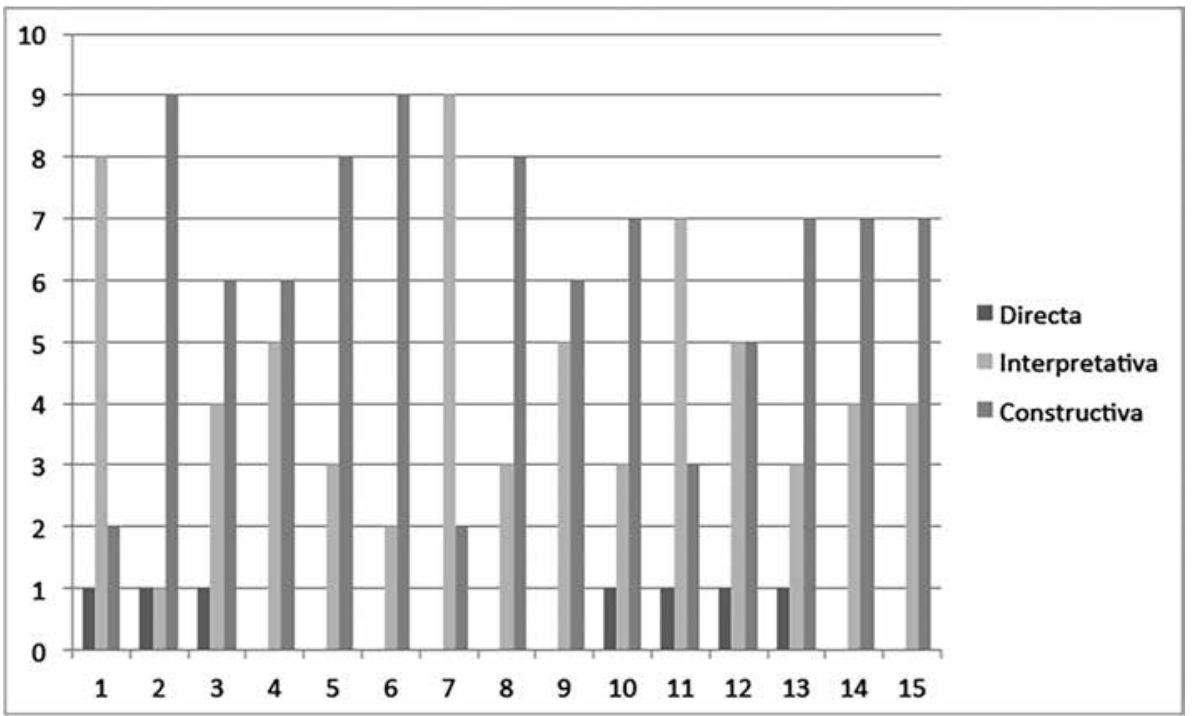

Sobre el instrumento para evaluar la acción de problematizar

A continuación se presentan los resultados correspondientes a la aplicación del cuestionario sobre la acción de problematizar. En primer lugar se examina la validez del instrumento, enseguida se realiza el análisis descriptivo de los resultados obtenidos.
El instrumento se construyó a partir de las seis características de la acción de problematizar expuestas en el marco teórico. Para cada una de estas características se elaboraron tres reactivos, como se explicó en el capítulo anterior. Los resultados de la prueba mostraron, según la aplicación del Coeficiente Alpha de Cronbach $=0,87$, una aceptable consistencia interna del cuestionario. Sin embargo, el aná- 
lisis factorial mostraba incoherencia respecto a algunos supuestos teóricos y los componentes encontrados. Por este motivo se presentan a continuación los resultados definitivos de la elaboración del instrumento de evaluación de la acción de problematizar, que es, además, uno de los objetivos propuestos en la presente investigación.

Los resultados correspondieron a la aplicación del cuestionario a 585 sujetos, distribuidos en 30 grupos, dos para cada profesor que participó en la investigación. En la aplicación del Coeficiente Alpha de Cronbach se obtuvo un valor de 0,855 , que continúa siendo un valor aceptable, con lo cual se establece una adecuada consistencia interna del instrumento. El valor de este coeficiente depende del número de reactivos y de sus covarianzas. Como el cuestionario consta de 18 ítems, el valor obtenido se puede considerar aceptable.

Los resultados del análisis de componentes principales, para analizar la validez de constructo, se presentan en la tabla 4, en la que se consideran los reactivos con carga superior a 0,3 para cada componente. Además, contiene una columna adicional, primera de la izquierda, que muestra las seis características de la acción de problematizar, a cada una de las cuales corresponden tres reactivos.

Se aprecia en esta tabla que el análisis factorial arroja tres componentes principales. Respecto a la primera característica se tiene que los reactivos 1 y 3 se encuentran en el segundo componente. Los reactivos 4 y 6 de la segunda característica también están en el segundo componente; asimismo, los reactivos 7 y 17. Los reactivos 9 y 12 de la tercera característica están en el tercer componente. Todos los reactivos de la cuarta característica se encuentran en el tercer componente. Todos los de la quinta están en el primer componente. Los reactivos 2 y 16 de la sexta característica se encuentran en el primer componente.
De acuerdo con el anterior análisis se considera que, al menos, dos reactivos de cada característica se encuentran en un mismo componente. Por tal razón, para efectos de la recolección de datos en función de determinar la acción de problematizar por parte de cada docente, se sacaran los promedios de los datos correspondientes a los reactivos de cada característica presentes en el mismo componente.

Según lo anterior, los reactivos que suman para evaluar cada característica quedan como sigue. A la primera característica, énfasis en los tres momentos de la acción de problematizar, corresponden los reactivos 1 y 3. Para la segunda, el alumno es considerado sujeto activo, reactivos 4, 6, 7 y 17 . Para la tercera, el problema como situación de impase, reactivos 9 y 12 . Para la cuarta, la praxis como dimensión ética de la acción, reactivos 8 y 10. Para la quinta, adquisición de estrategias de aprendizaje, reactivos 5,13 y 15 . Por último, para la sexta, cambio conceptual, reactivos 1 y 16.

Por otra parte, se observa, según los componentes obtenidos, tres grandes dimensiones. En la primera prevalecen las características 5 y 6; en la segunda, las características 3 y 4 , y en la tercera, las características 1 y 2 . Además, al analizar racionalmente las características, así como los reactivos presentes en cada componente, se infiere que el componente 1 se refiere principalmente a los aspectos cognitivos de la acción de problematizar; el componente 2, al carácter activo del alumno, y el componente 3 a los aspectos existencias y éticos. Este hallazgo resulta interesante en el sentido de indicar la posibilidad de considerar tres dimensiones de la acción de problematizar, con lo cual se facilita la comprensión de esta estrategia didáctica.

La tabla 5 muestra la proporción de varianza total explicada por cada factor. Los tres primeros factores agrupan los dieciocho dilemas del cuestionario, son capaces de explicar $43,066 \%$ de la variabilidad total, lo que se interpreta como un porcentaje aceptable. 
Tabla 4. Matriz de componentes rotada

\begin{tabular}{|c|c|c|c|c|c|}
\hline \multirow{2}{*}{\multicolumn{2}{|c|}{ Característica }} & \multirow{2}{*}{ Reactivos } & \multicolumn{3}{|c|}{ Componente } \\
\hline & & & 1 & 2 & 3 \\
\hline \multirow{3}{*}{1.} & \multirow{3}{*}{$\begin{array}{l}\text { Énfasis en los tres momentos de la acción de } \\
\text { problematizar. }\end{array}$} & 1 & & ,596 & \\
\hline & & 3 & 363 &, 594 & \\
\hline & & 17 &, 527 & ,352 & \\
\hline \multirow{3}{*}{2.} & \multirow{3}{*}{ El alumno es considerado sujeto activo. } & 4 & & 651 & \\
\hline & & 6 & &, 539 & \\
\hline & & 14 &, 544 & & \\
\hline \multirow{3}{*}{3.} & \multirow{3}{*}{ Problema como situación de impase } & 7 & & 644 & \\
\hline & & 9 & & & ,497 \\
\hline & & 12 & ,492 & & ,466 \\
\hline \multirow{3}{*}{4.} & \multirow{3}{*}{ La praxis como dimensión ética de la acción. } & 8 & & ,513 & ,483 \\
\hline & & 10 & & ,396 & ,597 \\
\hline & & 11 & & & ,716 \\
\hline \multirow{3}{*}{5.} & \multirow{3}{*}{ Adquisición de estrategias de aprendizaje. } & 5 & ,606 & & \\
\hline & & 13 & 597 & & \\
\hline & & 15 & 567 & & 343 \\
\hline \multirow{3}{*}{6.} & \multirow{3}{*}{ Cambio conceptual. } & 2 & ,467 & 367, & \\
\hline & & 16 & 600 & & \\
\hline & & 18 & & & 347, \\
\hline
\end{tabular}

Fuente: Elaboración propia.

Tabla 5. Varianza explicada por cada factor

\begin{tabular}{ccccccc}
\hline \multicolumn{5}{c}{ Valores iniciales } & \multicolumn{3}{c}{ Suma de la rotación de cargas } \\
\hline Componentes & Total & Varianza (\%) & Acumulado (\%) & Total & Varianza (\%) & Acumulado (\%) \\
\hline 1 & 5,403 & 30,018 & 30,018 & 2,876 & 15,978 & 15,978 \\
\hline 12 & 1,236 & 6,866 & 36,884 & 2,762 & 15,342 & 31,320 \\
\hline 4 & 1,113 & 6,182 & 43,066 & 2,114 & 11,745 & 43,066 \\
\hline 5 & 0,979 & 5,439 & 48,505 & & & \\
\hline 6 & 0,94 & 5,223 & 53,728 & & & \\
\hline 7 & 0,917 & 5,095 & 58,824 & & & \\
\hline 8 & 0,834 & 4,633 & 63,456 & & & \\
\hline 9 & 0,746 & 4,147 & 67,603 & & & \\
10 & 0,746 & 4,143 & 71,746 & & & \\
11 & 0,69 & 3,831 & 75,576 & & & \\
\hline 12 & 0,653 & 3,628 & 79,204 & & & \\
13 & 0,62 & 3,444 & 82,648 & & & \\
14 & 0,581 & 3,225 & 85,873 & & \\
15 & 0,559 & 3,108 & 88,981 & & \\
16 & 0,543 & 3,018 & 91,999 & & \\
17 & 0,529 & 2,938 & 2,554 & 94,938 & & \\
18 & 0,451 & 2,508 & 97,492 & & \\
\hline
\end{tabular}

Fuente: Elaboración propia. 


\section{Sobre la acción de problematizar}

La tabla 6 muestra la distribución de frecuencias de las evaluaciones de los profesores, discriminada por cada una de las características atribuidas a la acción de problematizar. Los resultados correspondientes a cada una de las seis características corresponden al promedio de los valores obtenidos en los ítems subyacentes a cada una de ellas. Se recuerda que para la calificación de cada reactivo se utilizó una escala ordinal en la que el valor 1 equivale a total desacuerdo con lo afirmado en el reactivo. 2 , en desacuerdo. 3, de acuerdo. 4, en total acuerdo.

Se observa una gran regularidad en los valores, salvo los datos de los profesores 7 y 8 , que obtuvieron valores inferiores a 3 en la tercera característica. Sin embargo, el promedio de todos los resultados, para cada docente, es superior a 3. Sobresalen el profesor 3, con el promedio más alto $(3,424)$, y el profesor 9 , con el promedio más bajo $(3,043)$. En todo caso, persiste la regularidad en los resultados, pues están por encima de tres y con una diferencia de 0,381 entre los valores extremos. Estos datos indican la tendencia de los profesores de la muestra a efectuar la acción de problematizar.

Los resultados obtenidos por cada docente ratifican el supuesto establecido en el análisis factorial del cuestionario, según el cual, los diversos reactivos guardan una gran cohesión. Según lo anterior, parece acertada la teoría asumida, en la que la acción de problematizar es una estrategia didáctica unitaria. Esta acción implica 6 características, las cuales, sin embargo, se refieren a un único fenómeno.

\section{Prueba de la hipótesis}

En esta investigación, cuyo objetivo principal consiste en establecer si hay o no asociación entre las teorías implícitas sobre la enseñanza y el aprendizaje, primera variable, y la acción de problematizar, segunda variable, se ha propuesto la hipótesis, según la cual sí hay relación. Por lo tanto, esta hipótesis se prueba si al correlacionar los datos correspondientes a las seis características de la acción de problematizar con los de las teorías implícitas resulta una correlación significativa, bien sea positiva o negativa.

Tabla 6. Distribución de las frecuencias según cada característica de la acción de problematizar

\begin{tabular}{|c|c|c|c|c|c|c|c|}
\hline & 1 & 2 & 3 & 4 & 5 & 6 & Promedio \\
\hline Profesor 1 & 3,201 & 3,209 & 3,067 & 3,154 & 3,209 & 3,507 & 3,225 \\
\hline Profesor 2 & 3,077 & 3,269 & 3,308 & 3,487 & 3,385 & 3,577 & 3,350 \\
\hline Profesor 3 & 3,402 & 3,440 & 3,163 & 3,536 & 3,406 & 3,598 & 3,424 \\
\hline Profesor 4 & 3,128 & 3,019 & 3,026 & 3,068 & 3,162 & 3,308 & 3,119 \\
\hline Profesor 5 & 3,242 & 3,189 & 3,152 & 3,293 & 3,232 & 3,379 & 3,248 \\
\hline Profesor 6 & 3,104 & 3,219 & 3,167 & 3,347 & 3,153 & 3,396 & 3,231 \\
\hline Profesor 7 & 3,083 & 3,215 & 2,986 & 3,102 & 3,250 & 3,486 & 3,187 \\
\hline Profesor 8 & 3,129 & 3,171 & 2,729 & 3,200 & 3,229 & 3,414 & 3,145 \\
\hline Profesor 9 & 3,021 & 3,016 & 3,042 & 3,042 & 3,014 & 3,125 & 3,043 \\
\hline Profesor 10 & 3,443 & 3,382 & 3,123 & 3,327 & 3,327 & 3,453 & 3,343 \\
\hline Profesor 11 & 3,162 & 3,213 & 3,059 & 3,255 & 3,304 & 3,309 & 3,217 \\
\hline Profesor 12 & 3,181 & 3,250 & 3,167 & 3,259 & 3,250 & 3,292 & 3,233 \\
\hline Profesor 13 & 3,256 & 3,128 & 3,038 & 3,137 & 3,239 & 3,308 & 3,184 \\
\hline Profesor 14 & 3,214 & 3,307 & 3,043 & 3,419 & 3,229 & 3,414 & 3,271 \\
\hline Profesor 15 & 3,277 & 3,213 & 3,053 & 3,149 & 3,206 & 3,457 & 3,226 \\
\hline
\end{tabular}

Fuente: Elaboración propia. 
En la tabla 7 se presentan los datos correspondientes a las dos variables, cuya correlación se analiza en este apartado. En primer lugar se consideran los valores correspondientes a la sumatoria de los datos obtenidos en cada una de las seis características de la acción de problematizar. En seguida se considera la sumatoria de los valores obtenidos en el cuestionario de dilemas.

Los valores asignados a cada dilema seleccionado fueron los siguientes: 10 , si correspon- día a la teoría directa. 20, a la interpretativa. 30, a la constructiva. Según lo expuesto en el marco teórico se considera que los docentes no tienen de manera exclusiva una teoría, sino que estas coexisten en el mismo sujeto. Además, puesto que se considera una continuidad que va de la teoría directa a la constructiva, se le atribuye más valor a esta última, de manera que la sumatoria de los valores obtenidos se incrementa en la medida que en el pensamiento del profesor prevalece la teoría constructiva.

Tabla 7. Datos sobre las características de la acción de problematizar y las teorías implícitas de la enseñanza y el aprendizaje

\begin{tabular}{cccccccc}
\hline \multicolumn{6}{c}{ Características de la acción de problematizar } & Teorías implícitas \\
\hline Profesor & 1 & 2 & 3 & 4 & 5 & 6 & \\
\hline 1 & 429 & 860 & 411 & 634 & 645 & 470 & 240 \\
\hline 2 & 80 & 170 & 86 & 136 & 132 & 93 & 300 \\
\hline 3 & 313 & 633 & 291 & 488 & 470 & 331 & 270 \\
\hline 4 & 244 & 471 & 236 & 359 & 370 & 258 & 270 \\
\hline 5 & 214 & 421 & 208 & 326 & 320 & 223 & 300 \\
\hline 6 & 149 & 309 & 152 & 241 & 227 & 163 & 310 \\
7 & 222 & 463 & 215 & 335 & 351 & 251 & 240 \\
\hline 8 & 219 & 444 & 191 & 336 & 339 & 239 & 300 \\
\hline 9 & 290 & 579 & 292 & 438 & 434 & 300 & 280 \\
\hline 10 & 365 & 717 & 331 & 529 & 529 & 366 & 280 \\
\hline 11 & 215 & 437 & 208 & 332 & 337 & 225 & 240 \\
\hline 12 & 229 & 468 & 228 & 352 & 351 & 237 & 260 \\
\hline 13 & 254 & 488 & 237 & 367 & 379 & 258 & 280 \\
\hline 14 & 225 & 463 & 213 & 359 & 339 & 239 & 290 \\
\hline 15 & 308 & 604 & 287 & 444 & 452 & 325 & 290 \\
\hline
\end{tabular}

Fuente: Elaboración propia.

La tabla 8 muestra los resultados del coeficiente de correlación de Spearman. Según se aprecia en esta tabla, solo existe correlación significativa y negativa entre las teorías implícitas y la característica 3 de la acción de problematizar, que corresponde a la presentación del problema como situación de impase. Esto, desde el punto de vista estadístico implica que en la medida que se aumenta la presencia de la teoría constructiva disminuye la presencia de la característica 3 y viceversa. 
Tabla 8. Análisis de las correlaciones

\begin{tabular}{lccccccc}
\hline & \multicolumn{6}{c}{ Acción de problematizar } \\
\hline \multirow{2}{*}{ Coeficiente rho de Spearman } & Teorías implícitas & 1 & 2 & 3 & 4 & 5 & 6 \\
& &,- 472 &,- 488 &,- 523 &,- 400 &,- 503 &,- 455 \\
\hline
\end{tabular}

Fuente: Elaboración propia.

Según los resultados obtenidos, se niega la hipótesis, pues solo se presenta la correlación significativa entre una característica de la acción de problematizar y las teorías implícitas de los docentes. Además, dicha correlación, considerando el tamaño de la muestra, tiende a no ser significativa. Se concluye, por tanto, que en esta muestra no existe evidencia estadísticamente significativa que permita afirmar la asociación entre las variables asociadas.

\section{Discusión}

Sobre la asociación entre las teorías implícitas sobre la enseñanza y el aprendizaje y la acción de problematizar

Los resultados que se acaban de presentar contradicen los presupuestos de las teorías implícitas sobre la enseñanza y el aprendizaje. A partir de Rodrigo et al. (1993) se tiene que las teorías implícitas constituyen un marco teórico general e implícito que fundamenta el quehacer del profesor. De ahí que fuese previsible la existencia de una correlación positiva entre la acción de problematizar, que tiene el carácter de estrategia didáctica, y las teorías implícitas de los docentes sobre la enseñanza y el aprendizaje. Pero, esta correlación no se verificó en la muestra estudiada.

Cabe, sin embargo, considerar que, aunque los resultados de la prueba estadística de la correlación no sean significativos, este hecho no conduce absolutamente a negar la existencia de asociación entre las variables. Aunque, para la muestra en cuestión se debe afirmar categóricamente que no hay evidencia estadística que permita afirmar la asociación entre las variables estudiadas.
La anterior consideración se apoya en los resultados obtenidos en los análisis descriptivos de los datos. Estos permiten sostener el supuesto de que existe asociación entre las variables. La teoría implícita más frecuente en la muestra fue la teoría constructiva. Esta apareció 92 veces, sobre 165 posibilidades, superando a las otras teorías (66 veces, la teoría interpretativa y 7, la teoría directa, ver tabla 4). Por su parte en la evaluación de la acción de problematizar, la media para cada uno de los seis atributos (características) fue superior a 3 , indicando una alta valoración de la acción de problematizar, como era de esperarse desde el punto de vista teórico, dado el telón de fondo constructivista de la problematización (Orange, 2005).

En la teoría implícita constructiva se considera que el alumno es sujeto activo en el proceso de construcción del conocimiento y aprender es una construcción antes que una copia del objeto disciplinar de conocimiento. En la acción problematizadora se considera que el docente antes que transmitir conocimientos, propone situaciones caracterizadas por ser una situación de impase, en otras palabras, el docente presenta situaciones en el aula de aprendizaje ante las cuales el alumno debe encontrar una solución, para lo cual debe buscar información y construir un conocimiento apropiado a la solución de la situación.

Los resultados obtenidos, al ser considerados a partir de las teorías, permiten establecer la asociación entre las teorías implícitas y la acción de problematizar. Inclusive, desde este punto de vista, se podría plantear que la acción de problematizar guarda una relación de dependencia con las teorías implícitas sobre la en- 


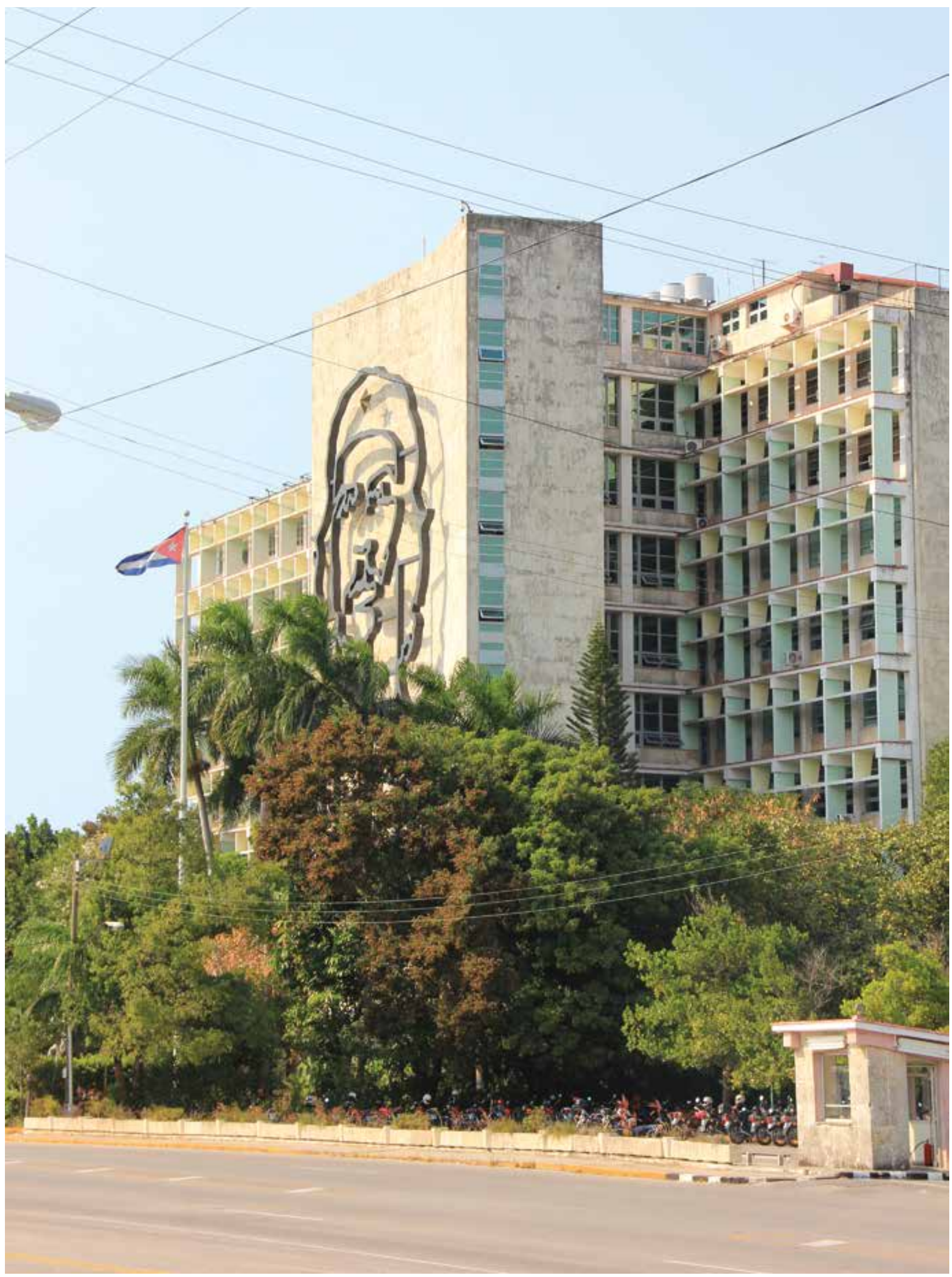


señanza y el aprendizaje, pues un docente en el que prevalece la teoría constructiva debería tender a realizar estrategias didácticas basadas en el constructivismo. Sin embargo, la prueba estadística no aporta ninguna evidencia sobre la asociación entre estas variables. ¿Cuál podría ser la explicación?

Responder a esta pregunta implica entrar en resbaladizo terreno de las hipótesis, de las suposiciones. Con todo, al revisar la naturaleza de las variables se encuentra una explicación que resulta razonable. Las teorías implícitas sobre la enseñanza y el aprendizaje no establecen una distinción tajante entre la teoría interpretativa y la teoría constructiva. Se supone que en un mismo sujeto pueden coexistir elementos de cada una de estas teorías (Pozo et al., 2006). Si se observan la figura 2 , se encuentra que respecto a la cuestión ¿Qué es aprender? prácticamente hay un empate entre la teoría directa y la teoría interpretativa. Asimismo, en la figura 3, se pueden encontrar, para algunos docentes, puntajes muy cercanos, incluso empates, en la frecuencia de la teoría interpretativa y la teoría constructiva. Esto indica la gran dificultad de verificar variaciones significativas en la suma total de los datos, pues los puntajes de la teoría interpretativa terminan afectando los valores totales.

A partir de esto se podría concluir que, aunque se observa de manera global la asociación entre la preferencia de la teoría constructiva y la tendencia a una valoración alta de la acción problematizar, en la prueba estadística esta correlación no aparece probablemente, porque las variaciones entre las puntuaciones no son suficientemente significativas. Respecto a la acción de problematizar caben consideraciones semejantes. Se partió del supuesto de que los docentes podrían tender a realizar en mayor o menor medida la acción de problematizar, pero los resultados obtenidos por todos los docentes tienden a ser altos. Con relación a los resultados obtenidos, en las dos variables se podría estar presentando una afectación conocida como restricción del rango, pues, ante una muestra pequeña homogénea, las correlaciones quedan comprometidas (Kantowitz, Roediger \& Elmes, 2001).

Queda abierta la necesidad de diseñar otras estrategias de evaluación, por ejemplo trabajar con variables categóricas, en lugar de ordinales, que exijan la caracterización de los docentes de acuerdo con sus teorías implícitas. Se podría diseñar otra estrategia de evaluación que no mida el grado de realización de la acción de problematizar, sino que establezca una distinción entre docentes que se caracterizan por asumir la problematización como estrategia típica y fundamental de su actividad en aula.

Por otra parte, puesto que la evaluación de la acción de problematizar fue efectuada por un evaluador externo, los estudiantes de cada profesor, cuando apenas habían transcurrido tres semanas del inicio de actividades académicas, se puede suponer que no haya un número significativo de cuestionarios respondidos al azar. Asunto sobre el que no se previó un control estadístico, el cual debería ser tenido en cuenta en futuras investigaciones de este tipo. Además, efectuar la evaluación al final del periodo lectivo, para disminuir las posibilidades de respuestas azarosas debidas a la escasa interacción con el docente, pues el evaluador califica de acuerdo con el menor o mayor conocimiento que tiene de la actividad del profesor.

En definitiva la evidencia estadística no permite afirmar la existencia de la correlación entre las variables estudiadas. Sin embargo, la interpretación de los datos sometidos al análisis descriptivo, apoyado en las afirmaciones del marco teórico, indica la existencia de la correlación. Ante tal ambigüedad se plantea la posibilidad de que diversos factores correspondientes al tratamiento de los datos sean la explicación del resultado estadístico inesperado. Entre tales factores se consideran: la naturaleza las medidas de las variables, la restricción del rango y las características del evaluador externo de la acción de problematizar. 


\section{Sobre el instrumento de evaluación de la acción de problematizar}

Se considera interesante abordar los datos referentes al instrumento diseñado para evaluar la acción de problematizar. En esta investigación se utilizó un concepto en el que se parte de la problematización como estrategia didáctica básica para que el proceso de aprendizaje se realice como una actividad en la que el aprendiz cumple un papel activo. Sin embargo, este concepto se presenta bajo el nombre de acción de problematizar para enfatizar con ello el papel del docente en cuanto agente que dispone condiciones que hacen posible el aprendizaje.

Se establecieron seis características de esta acción, a partir de las cuales se construyó un instrumento de evaluación. Al respecto se tiene que, aunque en el análisis factorial se encontraron tres componentes principales, en cada uno de ellos están presentes dos características, para un total de seis. Se trata de un dato evidenciado en el análisis estadístico, sobre el cuál se observa una interesante particularidad: las parejas de características que se encuentran en cada componente guardan entre sí importantes relaciones teóricas.

Se estableció que la acción de problematizar implica que se proponga o exponga a los alumnos ante una situación que moviliza un proceso constituido de tres pasos (primera características) en el que ellos son sujetos activos (segunda característica). Se podría decir que se trata de la dimensión operativa de la acción de problematizar, pues los pasos del proceso son efectuados por los estudiantes considerados actores del proceso de resolución. Los reactivos correspondientes a estas características se encuentran en el mismo componente.

La acción de problematizar tiene una dimensión existencial y ética. Esto es, la situación problemática es tal por afectar existencialmente al estudiante (tercera característica). La dimen- sión existencial como aparece en Freire (1983 y 2006) revela la condición humana del sujeto cognoscente. Al asumir una situación como problema, al buscar una solución, etc., está implicada la libertad del sujeto, por tanto su singular manera de estar en el mundo (esto es, de existir). En otras palabras, porque es importante para él encontrar una solución, superar el problema, y al hacerlo aparece el aspecto ético, pues no se trata simplemente de un juego intelectual, sino de una actividad que implica su manera de responder a los impases que se le presentan (cuarta característica). Estos dos aspectos se encuentran en el mismo componente hallado en el análisis factorial.

El tercer componente agrupa las características de tipo cognitivo, bien sea porque se trate de adquisición de nuevos conocimientos (sexta característica) o porque se refiere a la reflexión sobre el proceso de aprendizaje (quinta característica).

Esta agrupación de las seis características en tres componentes es un hallazgo no previsto en el diseño de la investigación. Sin embargo, como se acaba de exponer, la agrupación revelada por el análisis factorial se puede comprender desde el punto de vista teórico. Lo cual aumenta la validez teórica del instrumento, en el sentido de que las dimensiones encontradas guardan coherencia con la teoría. De este modo se podría suponer la unidad de la acción de problematizar, aunque reconociendo tres dimensiones que agrupan seis características.

Se trata de un dato considerable respecto a la construcción teórica sobre la problematización. Orange (2005) ha advertido la gran diversidad de marcos teóricos utilizados en la investigación sobre la problematización. Todos tienen como telón de fondo el constructivismo, pero hacen usos didácticos diversos del problema, al tiempo que este es asumido en diversidad de posturas epistemológicas. Sin embargo, en esta investigación, al definir las características de la problematización y, en consecuencia, de 
la acción de problematizar, se aunaron elementos del pragmatismo (Dewey), de la perspectiva crítico-histórica y existencialista (Saivani y Freire) y de la psicología cognitiva. Convergencia de referencias teóricas que parece posible de acuerdo con la unidad de evaluación detectada en el instrumento.

Sin embargo, los resultados sobre el instrumento de evaluación de la acción de problematizar sugieren que se vaya con cautela. Se deben considerar análisis adicionales referentes a la posibilidad de estar midiendo una única dimensión, como se podría deducir a partir del análisis de la varianza explicada por cada factor. Cabría cambiar el análisis factorial, en lugar de considerar los componentes principales, considerar solamente los que están explicando el 43,066\% acumulado. Además, se deberían hacer evaluaciones adicionales referentes a la validez de instrumento, por ejemplo, confrontar sus resultados con los obtenidos mediante otro tipo de evaluación.

En todo caso, no deja ser interesante la condensación de los componentes de la prueba, encontrada en el análisis factorial, y el hecho peculiar de que los ítems presentes en tales dimensiones se puedan agrupar en pares de características con afinidad teórica. Pero, es necesario depurar la evaluación de la confiabilidad y validez del instrumento mediante estudios adicionales, con el fin de reafirmar o descartar los interesantes hallazgos mencionados.

\section{Conclusiones}

Mediante la presente investigación se logró responder la cuestión principal, planteada en la definición del problema, la cual dice: ¿Existe una relación entre la acción de problematizar y los tipos de teorías implícitas sobre la enseñanza y el aprendizaje utilizados por los docentes del programa de Psicología? La solución es: no existe correlación entre la acción de problematizar y las teorías implícitas sobre la enseñanza y el aprendizaje, como quedó claramente establecidos en la prueba de la hipótesis.
Además de dar respuesta a la cuestión central de esta investigación, correspondiente a su objetivo principal, se pudieron alcanzar los objetivos específicos propuestos. Respecto a la evaluación del grado en que cada una de las teorías implícitas sobre la enseñanza y el aprendizaje está presente en cada profesor, los datos recogidos sobre su presencia en los docentes, permiten establecer cuál de estas tiene mayor presencia. La teoría implícita que prevalece es la constructiva. Esto es así en 12, de los 15 docentes participantes. Para dos de ellos, es la teoría interpretativa la que tiene mayor presencia. Y en uno de los docentes las teorías interpretativa y directa tuvieron resultados equitativos.

Puesto que no se encontró una correlación significativa entre las dos variables estudiadas, hay lugar a preguntar si "¿se podría suponer, desde el punto de vista teórico, que la acción de problematizar tiene una relación de dependencia respecto a las teorías implícitas?" Este continua siendo un asunto interesante para la elaboración teórica, tanto de parte de las teorías implícitas sobre la enseñanza y el aprendizaje, como del estudio de la problematización y la acción de problematizar.

El no haber encontrado evidencia estadísticamente significativa que permita afirmar la existencia, en los profesores de la muestra, de la asociación entre la acción de problematizar y las teorías implícitas sobre la enseñanzaaprendizaje contradice el supuesto de que el pensamiento del profesor afecta su actividad. Aunque, cabe considerar que la muestra es pequeña y no probabilística, con lo cual se limitan los alcances de los resultados. Por lo tanto, no se puede afirmar que los supuestos teóricos deban ser descartados, pero sí se puede afirmar que en una muestra estudiada la teoría no se cumplió. Lo que permite suponer que no siempre hay relación entre las teorías implícitas de los profesores y su actividad y que otros factores podrían conducir a que los docentes implementen esta estrategia didáctica, directrices institucionales, por ejemplo. 


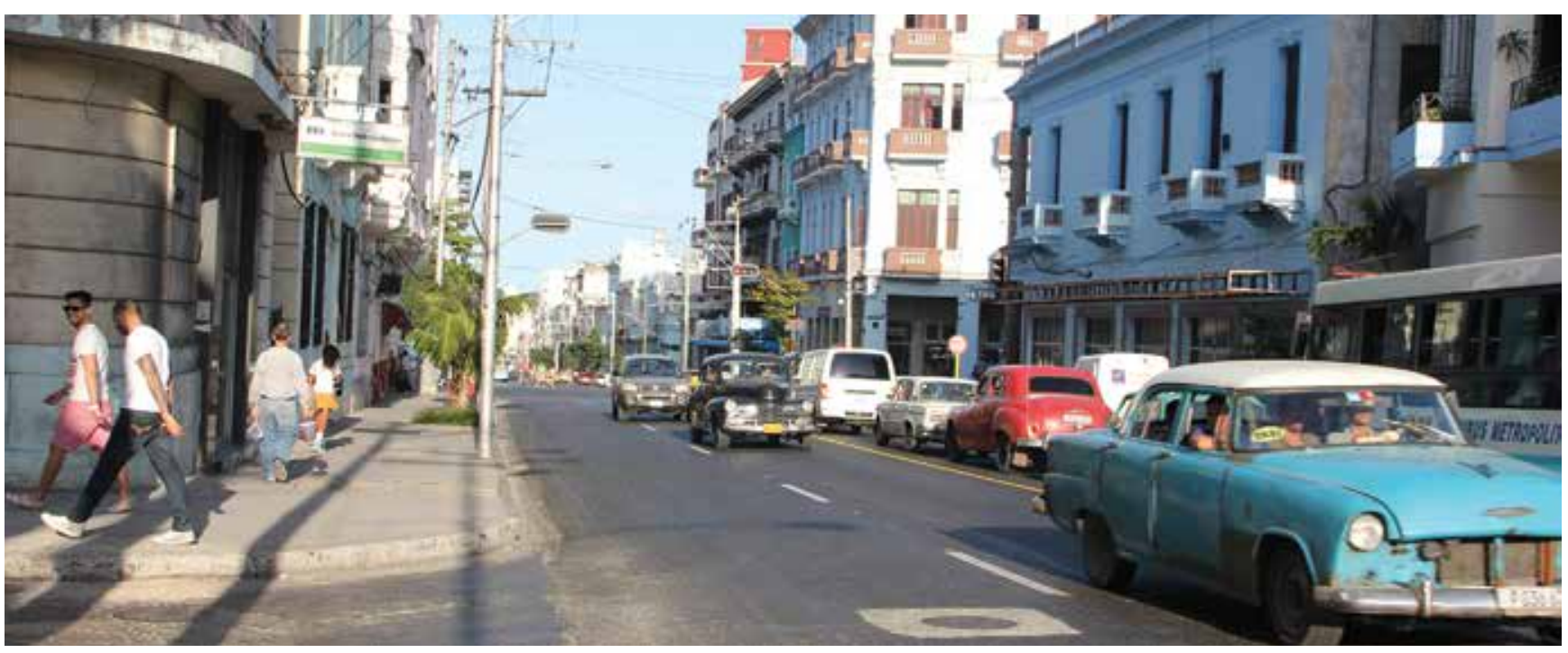

Por otra parte, en el proceso de construcción y aplicación del instrumento para evaluar la acción de problematizar por parte de los docentes, se encontró que las seis características de esta acción expuestas en la teoría se confirman en el análisis factorial de los componentes del cuestionario. Al respecto se tiene que, aunque en el análisis factorial se encontraron tres componentes principales, en cada uno de ellos están presentes dos características, para un total de seis. Sobre este dato se observa una interesante particularidad: las parejas de características que se encuentran en cada componente guardan entre sí importantes relaciones teóricas.

Respecto a las teorías implícitas sobre la enseñanza-aprendizaje, el análisis descriptivo de los resultados confirma las principales afirmaciones teóricas sobre el asunto. Los docentes tienen proporciones diversas de las teorías implícitas. Las teorías implícitas de los docentes tienden a tener una mezcla de la teoría interpretativa y constructiva, con poca presencia de la teoría directa.

El hecho de no haber encontrado una correlación estadísticamente significativa entre la acción de problematizar y las teorías implícitas sobre la enseñanza-aprendizaje da lugar a cuestionamientos acerca de las teorías y del método. Estas se enuncian a continuación.
- ¿En los docentes, priman otros factores, como las condiciones institucionales o el tiempo y el tipo de interacción establecida con los estudiantes, sobre las teorías implícitas sobre la enseñanza-aprendizaje, en la acción de problematizar?

- ¿En los estudiantes priman otros factores, como las condiciones institucionales o el tipo de interacción establecida con los docentes, en el momento de evaluar la acción de problematizar por parte de sus docentes?

- ¿Existe una relación de causa y efecto entre las teorías implícitas sobre la enseñanza-aprendizaje y la acción de problematizar?

En general se recomienda volver a abordar la misma pregunta de investigación, pero haciendo algunos ajustes en el método. La muestra debería ser probabilística, si se quiere que los resultados permitan conclusiones más amplias respecto a una población general de docentes o incluso sobre la teoría. Evidentemente, este es un asunto difícil, pues requiere recursos de tiempo y dinero, no siempre a la mano de los investigadores. En todo caso, aunque se trabaje con una muestra no probabilística la evaluación de la acción de problematizar, si se quiere utilizar el cuestionario elaborado en esta inves- 
tigación, se debe hacer al final de un periodo lectivo, en el que la realización de un recorrido con comienzo y final, ofrezca más precisión en la evaluación de acción de problematizar.

Aunque esta era una investigación cuantitativa, en cierto sentido, tenía un carácter exploratorio. Examinaba la existencia de una asociación sobre la cual no se encontró ningún estudio previo. En este caso se podrían hacer más estudios exploratorios, cuantitativos o cualitativos, antes de avanzar en el estudio sobre el tipo de relación entre las variables estudiadas. En estos casos se podría recurrir a otros instrumentos para la recolección de la información sobre los fenómenos estudiados.

Puesto que los estudios de las teorías implícitas sobre la enseñanza-aprendizaje sostienen que el pensamiento implícito del profesor afecta su actividad. En este caso se podría pensar en un estudio cuasi experimental, en el que se comparen las correlaciones existentes entre dos muestras, diferenciadas por el tipo de teoría implícita que caracteriza a los docentes. También es posible pensar en estudios cuasi experimentales en los que se pretenda determinar la diversa influencia de las variables que afecta la implementación de la acción de problematizar, por una parte, o la evaluación de la acción de problematizar llevada a cabo por los estudiantes, por otra.

Desde el punto de vista teórico sería muy valioso, mediante otros diseños investigativos, abordar el tema de las características o dimensiones de la acción de problematizar. Este asunto es importante, pues en la medida que se confirmen los hallazgos de esta investigación, se puede incrementar o disminuir el valor educativo de esta estrategia didáctica.

\section{Referencias}

Ausubel, D., Novak, J., \& Hanesian, H. (2006). Psicología educativa: Un punto de vista cognosctivo (2a. ed.). Distrito Federal, México: Trillas.
Chadwick, C. (2001). La psicología de aprendizaje del enfoque constructivista. Revista Latinoamericana de Estudios Educativos, 31(4), 111-126.

Fabre, M. (2005). Deux sources de l'épistémologie des problèmes: Dewey et Bachelard. Les Sciences de l'éducation - Pour l'Ére nouvelle, 3(38), 53-67. doi: 10.3917/lsdle.383.0053

Freire, P. (1983). Extensão ou comunicação? (7a. ed.). São Paulo: Paz e Terra.

Freire, P. (2006). Pedagogia da autonomia: saberes necessários (33a. ed.). São Paulo: Paz e Terra.

Kantowitz, B., Roediger, H., \& Elmes, D. (2001). Psicología experimental: cómo entender las investigaciones psicológicas ( $7^{\mathrm{a}}$ ed.). México: Thomson Learning.

Mariño, C. (2015). Problematizar: acción fundamental para favorecer el aprendizaje activo. Polisemia, (17), 40-54.

Orange, C. (2005). Problème et problematisation dans l'enseignement scientifique. Aster, (40), 3-10. Recuperado de http://ife.ens-lyon.fr/publications/edition-electronique/aster/RA040.pdf

Ormorond, J. (2008). Aprendizaje humano (4ª ed.). Madrid: Pearson Educación S.A.

Pérez, A. (1988). El pensamiento práctico del profesor/a. Implicaciones en la formación del profesorado. En Villa A. (ed.). Perspectivas y problemas de la función docente (pp. 128-149). Madrid: Narcea.

Pérez, A. (1983). Paradigmas contemporáneos de investigación didáctica. En Sacristán, G., y Pérez, A. (ed.). La enseñanza: su teoría y su práctica (pp. 95-138). Madrid: Akal.

Pérez, J., Mateos, M., Scheuer, N., \& Martín, E. (2006). Enfoques en el estudio de las concepciones sobre el aprendizaje y la enseñanza. En J. Pozo, N. Scheuer; M. Pérez, M. Mateos, E. Martín y M. Cruz. (Ed). Nuevas formas de pensar la enseñanza y el aprendizaje: Las concepciones de profesores y alumnos (pp. 55-94). Barcelona: Graó.

Pozo, J., Scheuer, N., Mateos, M., Pérez, M., \& Echeverria, M. (2006). Las teorías implícitas sobre el aprendizaje y la enseñanza. En J. Pozo, N. Scheuer, M. Pérez, M. Mateos, E. Martín y M. Cruz. (Ed). Nuevas formas de pensar la enseñanza y el aprendizaje: Las concepciones de profesores y alumnos (pp. 95-134). Barcelona: Graó.

Rodrigo, M., Rodríguez, A. \& Marrero, J. (1993). Las teorías implícitas: una aproximación al conocimiento cotidiano. Madrid: Visor.

Saviani, D. (1996). Educação do senso comum à consciência filosófica (11a. ed.). Campinas: Editora Autores Associados.

Vilanova, S., García, M. B., \& Señorino, O. (2007). Concepciones acerca del aprendizaje: diseño y validación de un cuestionario para profesores en formación. Revista Electrónica de Investigación Educativa, 9(2). Recuperado de http://redie.uabc.mx/vol9no2/contenido-vilanova.htm

Zanotto, M., \& Rose, T. (2003). Problematizar a própria realidade: análise de uma experiência de formação. Revista Educação e Pesquisa, 29(1), 45-54. Recuperado de http:// www.scielo.br/pdf/ep/v29n1/a04v29n1.pdf 


\section{Apéndice 1. Cuestionario de dilemas de Vilanova, García y Señorino}

En una reunión de departamento, los profesores están discutiendo acerca de distintos temas que tienen que ver con la enseñanza. Se exponen aquí los diferentes puntos de vista que aparecen. Le pedimos que señale la posición que representa mejor su opinión.

1. Con respecto al aprendizaje, algunos docentes opinan que:
a) Aprender es obtener la copia del objeto aunque algo distorsionada, debido al propio proceso de aprender.
b) Aprender es obtener la copia fiel de lo que se aprende.
c) Aprender es recrear el objeto de aprendizaje, necesariamente transformándolo.

2. Con respecto a las ideas previas de los alumnos, las principales opiniones fueron:
a) Son importantes fundamentalmente para el alumno, porque conocerlas le permite re- flexionar sobre sus propias ideas, contrastarlas con los modelos científicos y construir a partir de ellas su nuevo aprendizaje.
b) No es demasiado importante conocerlas, porque van a ser reemplazadas por los nuevos contenidos por aprender.
c) Es útil conocerlas, sobre todo para el docente, ya que le permite mostrarle al alumno la diferencia entre sus ideas y las de la ciencia, que son las correctas.

3. En relación con la extensión de los programas de las asignaturas, algunos docentes piensan que se debe:
a) Seleccionar los contenidos más adecuados para que los alumnos razonen y desarrollen estrategias de aprendizaje.
b) Enseñar todos los contenidos que surgen de la lógica disciplinar, ya que son indispensa- bles para que el alumno avance en la carrera.
c) Enseñar todos los contenidos que surgen de la lógica de la disciplina, sin descuidar que los alumnos razonen y comprendan lo más posible.

4. En cuanto a los objetivos principales de una asignatura son:
a) Procurar que los alumnos desarrollen estrategias que les permitan asignarle significado a lo que aprenden.
b) Procurar que los alumnos adquieran todos los conocimientos básicos fundamentales, ya que con el tiempo lograrán darles significado.
c) Procurar que los alumnos razonen y comprendan lo más posible, aunque no siempre lo logren en el caso de los contenidos más complejos.


5. En cuanto a las características fundamentales para tener en cuenta al seleccionar un libro de texto, los docentes opinaron que deben:

a) Presentar abundante y rigurosa información bien organizada.

b) Ofrecer variedad de actividades y problemas aunque no incluya todos los temas.

c) Destacar la información más importante y ofrecer actividades para el alumno.

6. Para que los alumnos aprendan a aplicar los conocimientos adquiridos, las opiniones fueron:

a) Enfrentarlos a situaciones cada vez más abiertas, donde el docente solo actúa como orientador.

b) Explicarles con claridad lo que deben hacer y plantearle unas cuantas situaciones similares para que practiquen lo que se les ha enseñado.

c) Explicarles con claridad cómo deben trabajar para luego ir enfrentándolos a situaciones diferentes.

7. Con respecto a la función del profesor, fundamentalmente es:

a) Explicar el tema por aprender y si el contenido lo permite, favorecer la discusión y el análisis.

b) Explicar en forma clara y acabada el saber establecido, tal como se lo acepta en la disciplina correspondiente.

c) Favorecer situaciones en las que el alumno desarrolle capacidades para realizar comparaciones, argumentar y desarrollar un pensamiento crítico respecto del tema por aprender.

8. En cuanto a los libros de texto que usan los alumnos, lo mejor es:

a) Que todos usen el mismo libro, para asegurarnos de que todos los alumnos aprendan lo mismo.

b) Que cada alumno cuente con diferentes fuentes de información: textos, papers, revistas de divulgación científica, etc. para poder contrastar diferentes opiniones y diversas perspectivas.

c) Que todos manejen el mismo texto, aunque sería bueno que el docente ofrezca en clase otros libros para hacer alguna consulta o comparar puntos de vista.

9. Respecto de cómo hacer preguntas para evaluar los aprendizajes, las opiniones fueron:

a) Las preguntas deben ser lo más concretas y claras posible, para que los alumnos no se dispersen en las respuestas.

b) Las preguntas deben ser lo más concretas y claras posible, pero a su vez permitirle al alumno llegar a la misma respuesta por distintos caminos. 
c) Las preguntas deben ser lo suficientemente abiertas como para que cada alumno pueda organizar su propia respuesta.

10. Respecto de las ventajas y los inconvenientes de hacer los exámenes permitiendo que los alumnos tengan el material de estudio delante, los docentes creen que:

a) No es una buena idea porque los alumnos no hacen el esfuerzo de estudiar los contenidos para la prueba.

b) Es una buena idea porque esto podría permitir valorar si los alumnos son capaces de utilizar la información disponible para elaborar su propia respuesta.

c) Puede ser una buena idea siempre y cuando se acompañe con alguna otra tarea que permita comprobar que el alumno conoce la información.

11. Al evaluar la resolución de un problema, lo más importante es:

a) Plantearle una situación problemática nueva e, independientemente del resultado final que obtenga, comprobar que puede ponderar distintos caminos y elegir entre una variedad de estrategias para resolverlo.

b) Plantearle una situación problemática similar a las trabajadas en clase y comprobar que el alumno sigue los pasos del procedimiento enseñado y llega al resultado correcto.

c) Plantearle una situación problemática nueva y comprobar que es capaz de seleccionar un procedimiento adecuado para llegar al resultado correcto. 


\section{Apéndice 2. Cuestionario para evaluar la acción de problematizar}

A continuación aparecen 18 afirmaciones referentes a las actividades del proceso de enseñanzaaprendizaje realizado en esta asignatura. Para cada una de estas afirmaciones, por favor, marque con una $\mathrm{X}$ en la casilla del frente según su grado de acuerdo o desacuerdo.

\begin{tabular}{lcccc}
\hline Afirmaciones & $\begin{array}{c}\text { En total } \\
\text { desacuerdo }\end{array}$ & $\begin{array}{c}\text { En } \\
\text { desacuerdo }\end{array}$ & De acuerdo & $\begin{array}{l}\text { Totalmente } \\
\text { de acuerdo }\end{array}$ \\
\hline
\end{tabular}

1. El profesor promueve que los alumnos identifiquen asuntos que requieren la búsqueda de una solución.

2. Las actividades desarrolladas en esta asignatura me han llevado a transformar mis conocimientos

3. El profesor promueve que los alumnos propongan soluciones a problemas sobre los cuales han investigado.

4. El profesor promueve que los alumnos usen sus conocimientos para abordar los problemas relacionados con los temas de estudio.

5. El reconocimiento de problemas y la búsqueda de una solución me hacen reflexionar sobre mi proceso de aprendizaje.

6. El profesor da lugar a que se debatan los conocimientos que los alumnos han adquirido sobre los temas estudiados.

7. El profesor da lugar a actividades en las que los alumnos reconocen situaciones que necesitan ser solucionadas.

8. Al proponer soluciones a los problemas abordados en la asignatura se considera a quienes se afecta con ello.

9. Usted considera que debe reunir información y trabajar para dar solución a los asuntos que han aparecido en la asignatura, porque si no lo hace, un problema importante para su vida se deja de lado.

10. El profesor les hace caer en la cuenta de las repercusiones sociales de los problemas y soluciones propuestas.

11. Los problemas vistos en la materia son importantes para mi contexto social.

12. Los asuntos tratados en clase hacen que usted se sienta con la necesidad de buscar información y dar alguna solución.

13. Considero que he mejorado en la búsqueda de soluciones a partir de mis estrategias de aprendizaje desarrolladas en la asignatura.

14. El profesor promueve que los alumnos busquen información por su propia cuenta.

15. Debido al reconocimiento de problemas y el trabajo de investigación para darles solución he mejorado mi manera de aprender.

16. A partir de las actividades desarrolladas en la asignatura he adquirido nuevos conceptos.

17. El profesor promueve que los alumnos busquen información para comprender los asuntos que aparecen problemáticos.

18. Reconozco que algunos conocimientos que traía al inicio de la asignatura no son suficientes para responder a las problemáticas que se me presentan. 